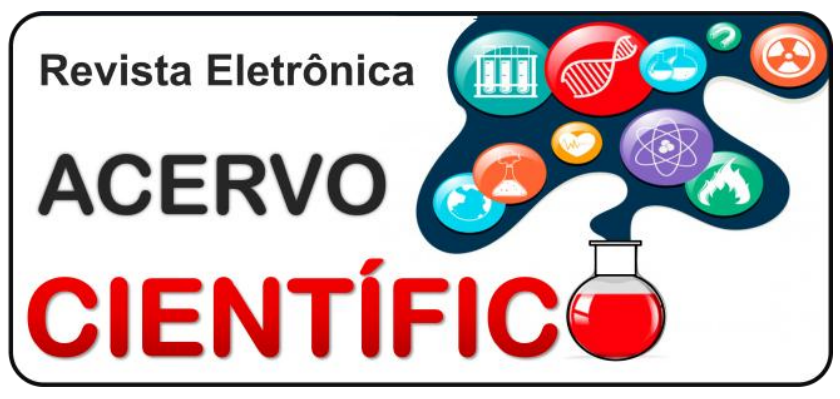

REVISÃO

Recebido em: 6/2019

Aceito em: 7/2019

Publicado em: 8/2019

\title{
Queixas osteomusculares do policiamento ostensivo em militares: revisão de literatura
}

\author{
Osteomuscular complaints of ostensive police in military: Review of literature
}

Quejas osteomusculares del policiamento ostensivo em militares: Revisión de la literatura

Meyrian Luana Teles de Sousa Luz Soares ${ }^{1 *}$, Daisy Karla Aroeira Tomaz da Silva ${ }^{2}$, Renata Inaly de Melo Silva².

Resumo: Os distúrbios osteomusculares relacionados ao trabalho acometem diversas atividades laborativas, na qual a classe dos militares tem sido bastante afetada em países desenvolvidos e, em desenvolvimento, como o Brasil. Para tal, o objetivo desse estudo é descrever as queixas osteomusculares do policiamento ostensivo em militares. Foi realizada uma revisão narrativa, com busca dos artigos indexados nas bases de dados MEDLINE via PUBMED e LILACS, no período entre fevereiro à junho de 2019, com os artigos publicados entre os anos de 2015 a 2019, utilizando os descritores "Osteomusculares", "Ergonomia", "Policiamento ostensivo" e "Militar" e seus correspondentes em inglês, utilizando o operador booleano AND. Foram excluídos artigos de revisão, artigos com abordagem cirúrgica e artigos experimentais com animais. Foram encontrados 367 artigos, dos quais 5 contemplaram os critérios de elegibilidade. Entre os achados, ressaltam-se alguns sintomas musculoesqueléticos na região lombar, cervical e joelhos, com a maior prevalência de quadro álgico e parestesias.

Palavras-chave: DORT, Ergonomia, Policiamento ostensivo.

\begin{abstract}
Work-related musculoskeletal disorders affect various work activities, in which the military class has been greatly affected in developed and developing countries such as Brazil. To this end, the aim of this study is to describe the musculoskeletal complaints of ostensive policing in the military. A narrative review was performed, searching the articles indexed in the MEDLINE databases via PUBMED and LILACS, from February to June 2019, with articles published from 2015 to 2019, using the descriptors "Osteomuscular", " Ergonomics"," Outstanding Policing "and" Military "and their English correspondents using the Boolean operator AND. Review articles, articles with surgical approach and experimental articles with animals were excluded. We found 367 articles, of which 5 met the eligibility criteria. Among the findings, we highlight some musculoskeletal symptoms in the lower back, neck and knees, with the highest prevalence of pain and paresthesias.
\end{abstract}

Keywords: DORT, Ergonomics, Overt policing.

\footnotetext{
1 Universidade Federal de Pernambuco (UFPE), Recife-PE. *E-mail: luanatsousa@gmail.com

${ }^{2}$ Centro Universitário Maurício de Nassau (UNINASSAU), Recife-PE.
} 
Resumen: Los trastornos musculoesqueléticos relacionados con el trabajo afectan diversas actividades laborales, en las que la clase militar se ha visto muy afectada en países desarrollados y en desarrollo como Brasil. Con este fin, el objetivo de este estudio es describir las quejas musculoesqueléticas de la policía ostensiva en el ejército. Se realizó una revisión narrativa, buscando los artículos indexados en las bases de datos MEDLINE a través de PUBMED y LILACS, de febrero a junio de 2019, con artículos publicados de 2015 a 2019, utilizando los descriptores "Osteomuscular", " Ergonomía "," Policía excepcional "y“ Militares "y sus corresponsales en inglés utilizando el operador booleano AND. Artículos de revisión, artículos con abordaje quirúrgico y artículos experimentales con animales fueron excluidos. Encontramos 367 artículos, de los cuales 5 cumplieron con los criterios de elegibilidad. Entre los hallazgos, destacamos algunos síntomas musculoesqueléticos en la parte baja de la espalda, cuello y rodillas, con la mayor prevalencia de dolor y parestesias.

Palabras clave: DORT, Ergonomía, Vigilancia policial.

\section{INTRODUÇÃO}

A relação entre o homem e a atividade laboral é amplamente discutida há anos. Em torno de $65 \%$ da vida do ser humano é dedicada ao trabalho (MAURO MYC, MUZI CD, GUIMARÃES RM, 2004). Diante dessa realidade, a participação de ciências que estudam as afecções e/ou desordens existentes em consequência desse tipo de atividade vem se mostrando significativa, uma vez que tais informações contribuem para o diagnóstico e a prevenção das lesões (AQUINO DS, QUEIROZ SJ, 2015; GOMES DM, SILVEIRA FGH, HORSTH AA, 2017).

Entre os fatores associados com uma maior predisposição a tais lesões podemos citar: o esforço físico, a postura inadequada, o controle rígido da produtividade, situações de estresse, jornadas de trabalho em períodos cumulativos, monotonia e a repetitividade (PENSRI P, JANWANTANAKUL P, CHAIKUMARN M, 2010).

As disfunções mais frequentemente encontradas, em torno de $50-65 \%$, pertencem ao grupo dos distúrbios osteomusculares relacionados ao trabalho (DORT), que podem estar associadas a outras comorbidades a depender do tipo de atividade exercida (SILVA JCP, PASCHOARELI LC, 2010; BERENGUER FA, SILVA DAL, CARVALHO CC, 2011; MESSING $\mathrm{K}$ et al., 2015).

O DORT vem sendo um dos grandes responsáveis pelo crescente absenteísmo no ambiente laboral, além do aumento no número de consultas médicas, internamentos, utilização de fármacos e/ou tratamentos complementares, representando altos índices nos custos de todos os envolvidos no processo, interferindo diretamente na relação empregado-empregador (PARK W et al., 2009).

Estudos mostram que, por utilizarem tratamentos de baixa eficácia, muitos trabalhadores desenvolvem doenças crônicas ou invalidez permanente (DETONI DJ, 2001; CASAROTTO RA; MENDES LF, 2018).

Há uma significativa relação entre trabalhadores que tem a permanência prolongada na postura em pé durante o desenvolvimento de suas atividades e os problemas no âmbito da saúde ocupacional (PENSRI P, JANWANTANAKUL P, CHAIKUMARN M, 2010).

Essa postura é desgastante e causa um alto grau de fadiga, pois mesmo estático, utilizamos a musculatura para sua manutenção. A musculatura é exigida de forma moderada, mas o seu uso prolongado causa desconforto muscular, como dores nos membros inferiores e na coluna vertebral (FERREIRA DKS, BONFIM C, AUGUSTO LGS, 2011).

A exposição aos riscos e à integridade física é evidente em determinadas categorias profissionais, como nos militares, em especial aqueles que praticam o policiamento ostensivo (SANTOS MMA, SOUZA EL, BARROSO BIL, 2017). Devido a características peculiares de sua atividade laboral, como a manutenção postural ereta, além de longas caminhadas e/ou corridas com equipamentos pesados como o colete 
balístico e cinto de guarnição, ou até o uso exaustivo da força muscular estão associados ao quadro de fadiga e dor, tornando esse tipo de profissão exposta diretamente aos riscos ocupacionais (FERREIRA DKS, BONFIM C, AUGUSTO LGS, 2011; MACEDO BF, 2017). Sendo assim, o objetivo desse estudo é identificar, através de evidências científicas, a frequência de queixas ocupacionais relacionada ao policiamento ostensivo em militares.

\section{MÉTODOS}

Foi realizada uma revisão narrativa da literatura através das bases de dados Literatura Latino-Americana e do Caribe em Ciências da Saúde (LILACS) e Medical Literature Analysis and Retrieval System on-line (MEDLINE) via PUBMED, utilizando os descritores em Ciências da Saúde - DeCS "Osteomusculares", "Ergonomia", "Policiamento ostensivo" e "Militar" e seus correspondentes em inglês Cumulative Trauma Disorders, Ergonomics, Ostensible policing Hospitals e Military, no período entre fevereiro à junho de 2019, com os artigos publicados entre os anos de 2015 a 2019, utilizando o operador booleano AND. Os descritores foram combinados segundo descrição no Quadro 1.

Quadro 1 - Estratégia de busca entre os descritores para a busca dos artigos.

\begin{tabular}{|l|l|}
\hline Base de dados & Descritores e títulos \\
\hline MEDLINE & Vumulative Trauma Disorders AND Ergonomics \\
PUBMED & Cumulative Trauma Disorders AND Military \\
& Cumulative Trauma Disorders AND Ostensible policing Hospitals \\
& Ergonomics AND Military \\
& Ergonomics AND Ostensible policing Hospitals \\
& Cumulative Trauma Disorders AND Ergonomics AND Ostensible policing \\
& Hospitals \\
\hline LILACS & Osteomusculares AND Ergonomia \\
& Osteomusculares AND Militar \\
& Osteomusculares AND Policiamento ostensivo \\
& Ergonomia AND Militar \\
& Ergonomia AND Policiamento ostensivo \\
& Osteomusculares AND Policiamento ostensivo AND Ergonomia \\
& Osteomusculares AND Ergonomia AND Militar \\
\hline
\end{tabular}

Fonte: Dados do autor, 2019.

Os artigos foram incluídos por: artigos indexados nas bases de dados selecionadas, com textos completos e gratuitos, publicadas nos períodos de 2015 a 2019 nos idiomas inglês, espanhol e português, além de contemplarem o tema proposto. Foram excluídos artigos de revisão, artigos com abordagem cirúrgica e artigos experimentais com animais.

Para a seleção dos artigos utilizou-se como base a estratégia do The Prisma Statement (Figura 1). A tabulação e análise dos dados foram realizadas de forma descritiva, utilizando uma tabela contendo variáveis que responderam à questão norteadora desta revisão. Os tópicos de interesse foram: autor e ano, objetivos, instrumentos utilizados e resultados. Logo após leitura na íntegra dos artigos incluídos, foram descritos o que cada artigo trazia que respondesse à pergunta norteadora, possibilitando observar, descrever e classificar os dados, com intuito de reunir o conhecimento produzido sobre o tema explorado na revisão.

\section{RESULTADOS E DISCUSSÃO}

A busca gerou um total de 367 referências. Com a exclusão das duplicatas, restaram 365 referências. Após a aplicação dos critérios de elegibilidade, 5 artigos foram selecionados para esta revisão. Entre os principais resultados encontrados, a presença de sintomas osteomusculares na população estudada de forma unânime, sendo a coluna lombar a região em que os militares sentem mais dores, além da região cervical e joelhos. Entre as manifestações clínicas predominantes, a dor e a parestesia foram os mais prevalentes, conforme os estudos descritos na Tabela 1. 
Figura 1 - Fluxograma da estratégia de busca e seleção dos artigos.

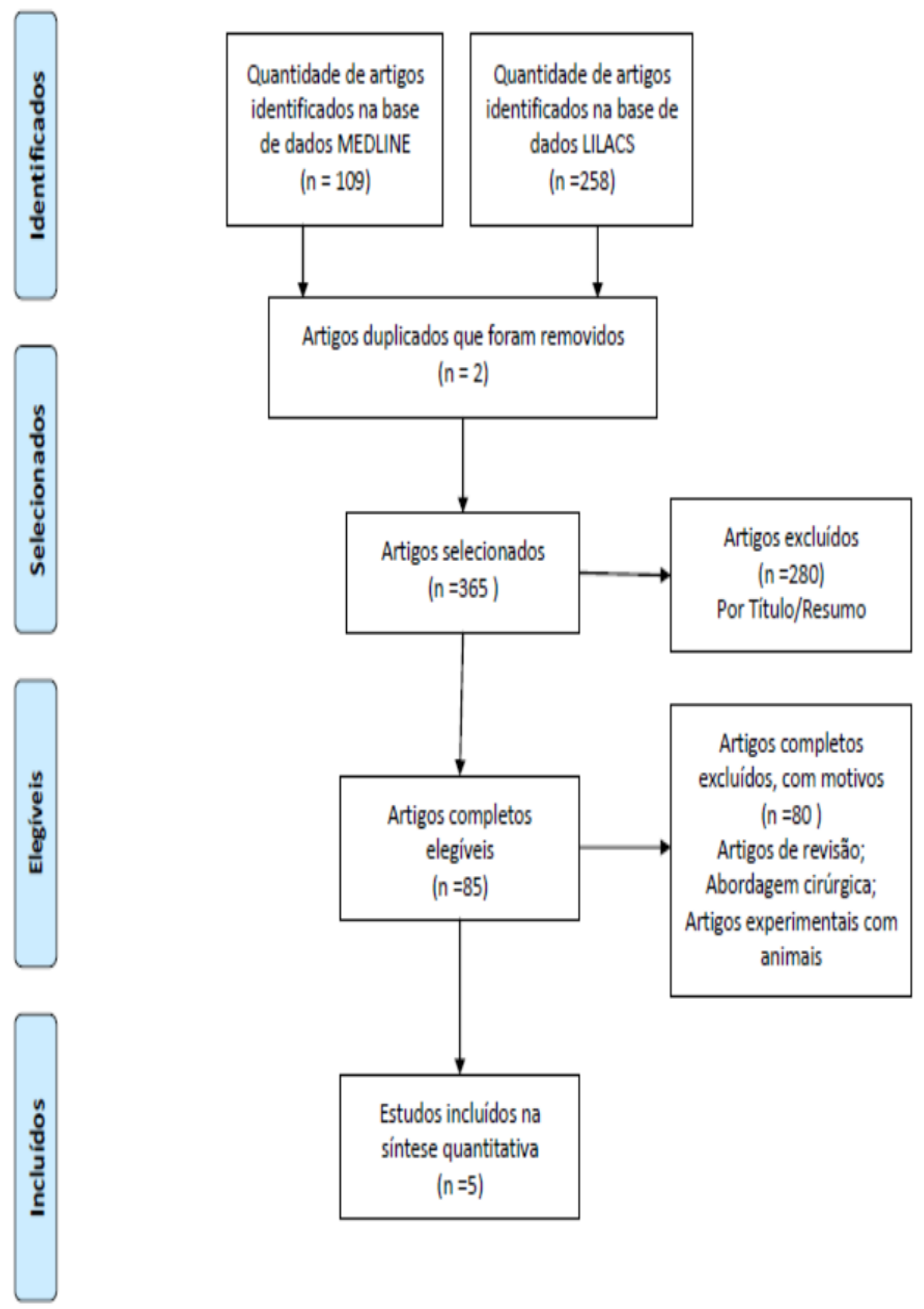

Fonte: The Prisma Statement (2019). 
Tabela 1 - Distribuição dos artigos de acordo com o título, autores/ano, objetivos e resultados.

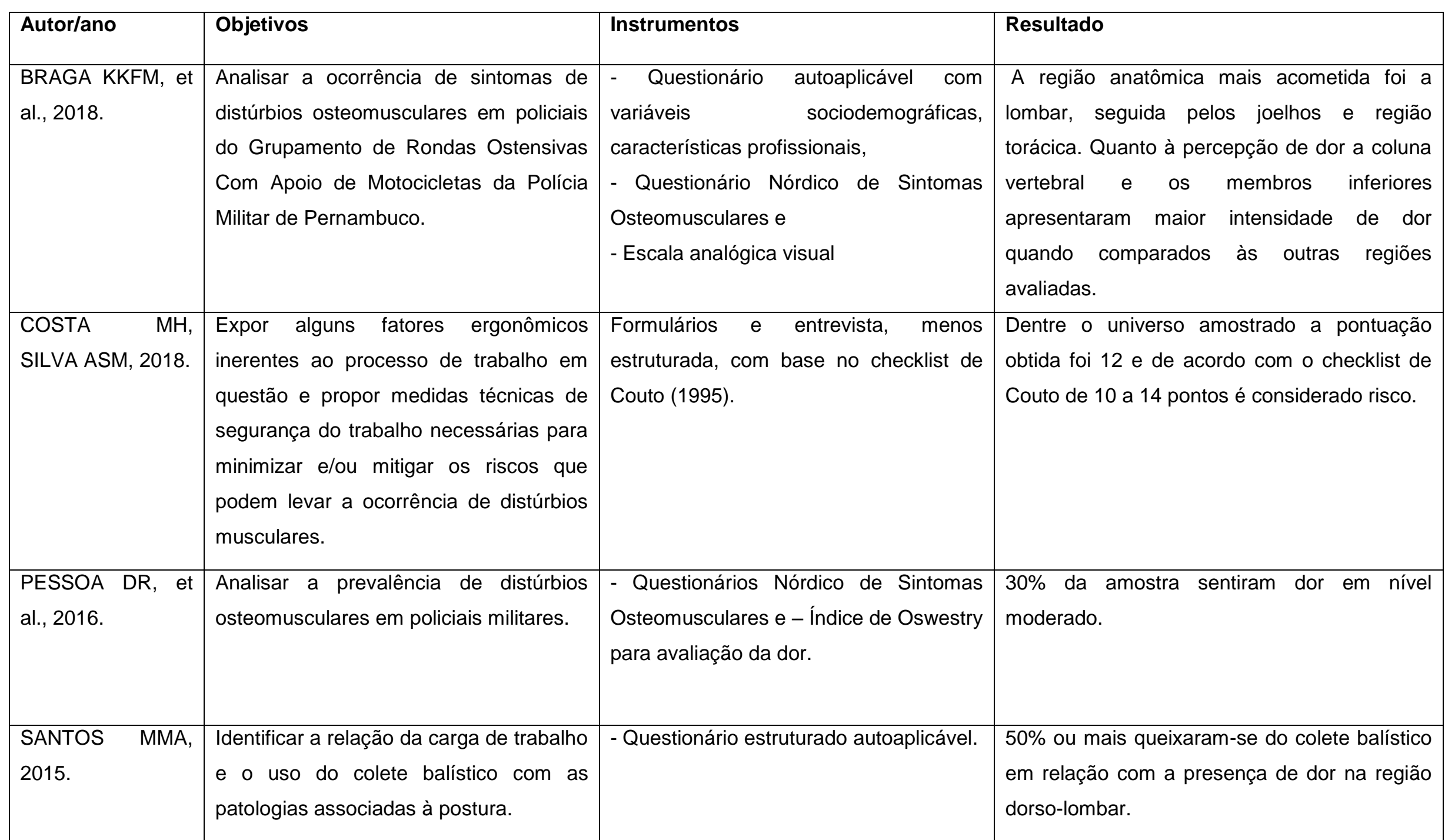

Fonte: Dados do autor, 2019. 
Os achados de Braga KFM et al (2018) corrobora com os estudos de Pessoa DR, et al., (2016), Santos MMA (2017) e Santos MC (2016) quanto aos sintomas musculoesqueléticos apontados pelos militares, destacando-se a parte inferior das costas (região lombar), dorso, cervical e joelhos com a maior prevalência de dor, porém a região lombar apresenta maior incidência de manifestações clínicas como dor, formigamentos e dormências, diferente dos achados de Costa MH, Silva ASM (2018).

As dores na coluna estão entre os agravos osteomusculares mais frequentes nessa população conforme descrevem os estudos supracitados. Santos MC (2016) associa a sobrecarga dos equipamentos utilizados pelos militares durante o trabalho com a presença do quadro álgico. Braga KFM et al (2018) quando o trabalho muscular estático for acrescido a carga dos coletes balísticos, aumenta a propensão e indução mais rápida à fadiga e às lesões das estruturas do sistema musculoesquelético, corroborando com os achados de Santos MC (2016), acerca da utilização do material adicional ao serviço.

De acordo com Braga KFM et al (2018), a dor não foi considerada uma causa de limitação para o desempenho das atividades profissionais e da vida diária. Porém, Santos MC (2016) e Santos MMA (2017) demonstram que os militares estão em constantes riscos que podem gerar problemas físicos, como distúrbios osteomusculares, ocasionando desconfortos, influenciando diretamente na qualidade de vida destes profissionais de forma negativa, sendo alvo em futuros estudos da queda no rendimento e no absenteísmo laboral.

\section{CONSIDERAÇÕES FINAIS}

A identificação das principais queixas presentes no policiamento ostensivo em militares, como as desordens na coluna vertebral e em articulações periféricas, foi associada a manifestações clínicas como dor e parestesias. Tais achados poderão servir como base para implementar alterações na rotina laboral, contribuindo para a prevenção de novas queixas. A investigação de outras desordens, como cardiovasculares e psicossomáticas, parece ser um bom ponto de partida para novas pesquisas com 0 público em questão.

\section{AGRADECIMENTOS E FINANCIAMENTO}

O estudo em questão não apresentou nenhum tipo de financiamento; gostaríamos de agradecer a Pósgraduação em Saúde da Criança e do Adolescente (UFPE - Vínculo do orientador) e ao Centro Universitário Maurício de Nassau (UNINASSAU) pela disponibilidade e contribuição no desenvolvimento do manuscrito.

\section{REFERÊNCIAS}

1. AQUINO DS, QUEIROZ SJ. Saúde do trabalhador na construção civil: medidas preventivas. Bahia. Monografia (Especialização em Enfermagem do Trabalho) - Escola de Medicina e Saúde Pública, 2015.

2. BERENGUER FA, SILVA DAL, CARVALHO CC. Influência da posição ortostática na ocorrência de sintomas e sinais clínicos de venopatias de membros inferiores em trabalhadores de uma gráfica na cidade do Recife-PE. Rev Bras de Saúde Ocupacional. n.36, v.123, p.153-161, 2011.

3. BRAGA KKFM, SOUZA FT, SKAPEC MVC, et al. Dor e desconforto musculoesquelético em policiais militares do Grupamento de Rondas Ostensivas com Apoio de Motocicletas. Rev Bras JP. n.1, v.1, 2018.

4. CASAROTTO RA, MENDES LF. Queixas, doenças ocupacionais e acidentes de trabalho em trabalhadores de cozinhas industriais. Rev Bras. de Saúde Ocupacional. n.28, v.107, p.119-126, 2018.

5. COSTA MH, SILVA ASM. Fatores ergonômicos que podem interferir na qualidade de vida dos policiais militares e podem gerar distúrbios musculares. Biblioteca digital de Segurança Pública. Goiás, 2018.

6. DETONI DJ. Estratégias de avaliação da qualidade de vida no trabalho: estudos de casos em agroindústrias. Santa Catarina. Dissertação (Mestrado em Engenharia de Produção) - Universidade Federal de Santa Catarina, Programa de Pós-Graduação em Engenharia de Produção, 2001.

7. FERREIRA DKS, BONFIM C, AUGUSTO LGS. Fatores associados ao estilo de vida de policiais militares. Ciência e Saúde Coletiva. n.16, v.8, p. 3403-3412, 2011.

8. GOMES DM, SILVEIRA FGH, HORSTH, AA. Avaliação ergonômica do trabalhador na construção civil: riscos minimizados por regulamentação e ginástica laboral. Meio Ambiente e Saúde. n.7, v.1, p.17-27, 2017. 
9. MACEDO BF. Associação entre dor cervical e no ombro em relação ao estresse emocional. Brasília. Dissertação (Mestrado em Ciências da Saúde) - Universidade de Brasília, 2017.

10. MAURO MYC, MUZI CD, GUIMARÃES RM. Risco ocupacionais em saúde. Rev Enferm UERJ. V. v.12, p.338-45, 2004.

11. MESSING K, STOCK S, CÔTÉ J, et al. Is sitting worse than static standing? How a gender analysis can move us toward understanding determinants and effects of occupational standing and walking. J occup environ hyg. n.12, v.3, p.11-17, 2015.

12. PARK W, SINGH, DP, LEVY MS, et al. Obesity effect on perceived postural stress during static posture maintenance tasks. Ergonomics. n.52, v.9, p.1169-1182, 2009.

13. PENSRI $P$, JANWANTANAKUL P, CHAIKUMARN M. Biopsychosocial Risk Factors for Musculoskeletal Symptoms of the Spine in Salespeople. Int j occup environ health. n.16, v.3, p.303-311, 2010.

14. PESSOA DR, DIONISIO AG, LIMA LDV, et al. Incidência de distúrbios musculoesqueléticos em policiais Militares pelo impacto do uso de colete balístico (Apresentação na XX Encontro Latino Americano de Iniciação Científica e VI Encontro de Iniciação à Docência - Universidade do Vale da Paraíba, 2016.

15. SANTOS MMA. A influência do uso do colete balístico nas patologias relacionadas a postura em policiais militares. Repositório da Universidade Federal da Paraíba, 2015.

16. SANTOS MC. Análise eletromiográfica da sobrecarga postural causada pelo colete balístico em profissionais de segurança pública. Paraná. Dissertação [Mestrado em Engenharia Biomédica] - Universidade Tecnológica Federal, 2016.

17. SANTOS MMA, SOUZA EL, BARROSO BIL. Análise sobre a percepção de policiais militares sobre o conforto do colete balístico. Fisioter Pesqui. n.24, v.2, p.157-162, 2017.

18. SILVA JCP, PASCHOARELI LC. A evolução histórica da ergonomia no mundo e seus pioneiros. Cultura acadêmica. v.13, 2010. 